27 | 2001

Histoire de l'enseignement du français langue

étrangère ou seconde dans le bassin méditerranéen.

Volume 1

\title{
De Leo Spitzer à A.-J. Greimas La création du département de langue et littérature françaises de la Faculté des Lettres de l'Université d'Istanbul
}

\section{Osman Senemoglu}

\section{OpenEdition \\ Journals}

\section{Édition électronique}

URL : https://journals.openedition.org/dhfles/2593

DOI : $10.4000 /$ dhfles.2593

ISSN : 2221-4038

Éditeur

Société Internationale pour l'Histoire du Français Langue Étrangère ou Seconde

Édition imprimée

Date de publication : 1 décembre 2001

ISSN : 0992-7654

\section{Référence électronique}

Osman Senemoglu, « De Leo Spitzer à A.-J. Greimas La création du département de langue et littérature françaises de la Faculté des Lettres de l'Université d'Istanbul », Documents pour l'histoire du français langue étrangère ou seconde [En ligne], 27 | 2001, mis en ligne le 31 janvier 2014, consulté le 27 mai 2021. URL : http://journals.openedition.org/dhfles/2593 ; DOI : https://doi.org/10.4000/dhfles. 2593

Ce document a été généré automatiquement le 27 mai 2021 


\title{
De Leo Spitzer à A.-J. Greimas La création du département de langue et littérature françaises de la Faculté des Lettres de l'Université d'Istanbul
}

\author{
Osman Senemoglu
}

1 L'actuel département de langue et littérature françaises de la Faculté des Lettres de l'Université d'Istanbul fut fondé en 1933, sous la direction du grand romaniste germanophone Leo Spitzer (1887-1960), qui, quelques années plus tard, confia son poste à un autre savant allemand, Erich Auerbach (1892-1957). Mais il faut signaler que cette création fut réalisée dans des conditions bien singulières.

2 Au début de cette période, comme chacun sait, la montée du nazisme en Allemagne commençait à inquiéter les partisans de la démocratie et tout particulièrement la communauté juive vivant dans ce pays.

3 A la même époque, en Turquie, le président de la république Mustafa Kemal Atatürk prit l'initiative d'une réforme universitaire afin de moderniser l'enseignement supérieur et de supprimer les approches pseudo-scientifiques et les traditions théologiques.

4 Tenant compte de cette situation tragique qui se développait en Allemagne, le gouvernement turc lança un appel aux savants allemands d'origine juive. Un professeur suisse, Albert Malche, qui enseignait la pédagogie à l'Université de Genève, était alors le conseiller du gouvernement turc. C'est lui qui établissait les relations entre les autorités turques et les savants étrangers désirant immigrer en Turquie. Ainsi, des Allemands opposants au nazisme ou des Juifs, des médecins comme Erich Frank, des économistes comme Fritz Neumark, Gerhard Kessler, des philosophes comme Hans Reichenbach, Ernst von Aster, l'orientaliste Clemens Bosch, les juristes Andreas Schwartz et Ernst Hirsch, le pédagogue Wilhelm Peters, le compositeur et musicologue 
Paul Hindemith, le géologue Wilhelm Salomon-Calvi, le botaniste Hans Bremer, et j'en passe, vinrent travailler dans les universités turques à partir de 1933.

C'est dans ces circonstances que Léo Spitzer arriva à l'Université d'Istanbul. On lui demanda de créer un département de littérature occidentale au sein de la Faculté des lettres. Mais Spitzer refusa cette proposition, disant qu'il n'était pas spécialiste en la matière, qu'il était « romaniste ». C'est ainsi qu'il fonda l'Institut de romanologie ${ }^{1}$.

Dans l'équipe de Spitzer figuraient des noms comme :

- Herbert Dieckmann, spécialiste de Diderot (il partit aux États-Unis en 1937 et travailla dans le département de romanologie des universités de Saint-Louis, Harvard et Cornell);

- Lieselotte Dieckmann (épouse de Herbert Dieckmann, spécialiste de littérature comparée);

- Hans Marchand (qui travailla en Turquie jusqu'en 1953 et partit ensuite à l'université de Yale avant de retourner en 1957 à Tübingen où il enseigna la littérature anglaise);

- Eva Buck (qui travailla dans le département de romanologie jusqu'aux années 60 avant de s'installer en France. On lui doit une Introduction à la littérature occidentale pour les étudiants turcs).

7 Toujours dans cette même équipe figuraient comme lecteur un Italien, Bartalini, ainsi qu'une allemande Rosemarie Burkart, étudiante de Spitzer et assistante de Malche.

La seconde tâche de Spitzer à l'Université d'Istanbul fut la création d'une École des langues étrangères qui visait à enseigner une langue occidentale à tous les étudiants de l'université afin qu'ils puissent suivre les nouvelles publications scientifiques parues dans leur discipline. Inutile de dire que, jusqu'aux années 50, le français était la première langue choisie par les étudiants. Outre cette langue, étaient enseignés l'allemand, l'anglais ainsi que l'italien et le russe.

Quant à Erich Auerbach, qui travaillait, comme Spitzer, à l'Université de Marburg depuis 1929, il fut licencié en 1935 et s'exila, lui aussi, pour arriver à Istanbul en 1936 où il resta jusqu'en 1947 avant de partir aux États-Unis. C'est lors de son séjour à Istanbul qu'il écrivit Mimesis et Introduction à la philologie romane.

10 Après avoir résumé les conditions dans lesquelles ces grands savants se sont exilés, je voudrais dire quelques mots sur leur apport à l'étude de la littérature française en Turquie.

11 Force est de signaler que cet Institut de romanologie et cette Ecole des langues étrangères n'ont pas été créés ex nihilo : ils sont issus :

d'une double et longue tradition : une tradition de culture française, profondément enracinée en Turquie depuis plusieurs siècles, grâce à une présence constante de la France, à des échanges permanents et à une étroite parenté d'esprit et de cœur entre ces deux pays ; une tradition aussi de large humanisme, qui, tout en respectant les valeurs fondamentales de l'âme et de la nation turques, a fait de la Turquie, à travers les âges, un véritable carrefour de civilisations et de cultures, et lui a signifié de longue date sa vocation de conciliatrice et d'actif témoin. Cette double tradition, la Faculté des Lettres de l'Université d'Istanbul l'a recueillie, et singulièrement son Institut de Philologie française et romane, à la tête duquel se sont succédés des hommes de haute science comme les professeurs Spitzer, Auerbach et Trahard, qui ont su, peu à peu, s'entourer d'une équipe de collaborateurs compétents et dévoués ${ }^{2}$.

Ainsi naquit une tradition d'enseignement et de recherche basée sur l'analyse des textes littéraires, qui se réalise par des séminaires et des analyses philologiques. 
13 Mais il faut signaler que l'enseignement spécifique de la littérature française dans le cadre d'une chaire universitaire commence avec Spitzer. Auparavant, on y enseignait la littérature occidentale d'une manière hétéroclite. Cet enseignement n'avait, en effet, aucune méthode. On passait ainsi de Dante à Tolstoï, et de Balzac à Goethe. On faisait en quelque sorte une histoire littéraire élaborée selon les lectures des enseignants, et les cours étaient en turc.

Lorsque Spitzer fonda cet Institut de romanologie, la langue utilisée pour l'enseignement fut le français. Les premières étudiantes, au nombre de trois, furent des jeunes filles (une en 1933 et deux en 1934) frâichement diplômées des écoles françaises d'Istanbul gérées alors par des religieuses.

Juif autrichien, nourri d'humanités classiques, Spitzer, évoquant son apprentissage du français, cite au début d'une de ses études la phrase de Valéry Larbaud : « ... cette langue, je l'ai apprise comme l'on obtient l'amour d'une femme » (cité dans Starobinski $1970: 14)$.

16 Cette langue apprise avec passion lui servit d'instrument de communication pour transmettre son savoir dans un pays tiers. C'était une première que cette utilisation du français comme langue d'enseignement dans les études supérieures.

Mais c'est au niveau méthodologique et théorique que se situa la plus grande contribution de Spitzer et, par la suite, de Auerbach. Grâce à eux, l'approche scientifique des textes littéraires, soutenue par les données de la linguistique moderne, fut ancrée dans le département en question.

18 Je voudrais vous rappeler brièvement le point de vue de Spitzer. D'après lui « la stylistique doit combler l'intervalle séparant l'histoire littéraire et la linguistique. " Pour ce faire, sa méthode consistait à " apercevoir un écart stylistique par rapport à l'usage moyen ; - évaluer cet écart, qualifier sa signification expressive ; - concilier cette découverte avec le ton et l'esprit général de l'œuvre ; à partir de là, - définir plus amplement le caractère spécifique du génie créateur et, à travers lui, une tendance de l'époque » (1970 : 19) : tel est le mouvement que s'assigne au départ la critique spitzerienne.

En partant de cette approche, on pourrait placer Spitzer parmi les précurseurs de la sémiolique et de la critique structuraliste.

Dans les années 60, lors de ses conférences à la Faculté des lettres, Roland Barthes ne cachait pas son admiration pour le savant autrichien et le considérait comme l'un de ses maîtres à penser.

La troisième activité majeure à laquelle se consacra Spitzer à l'université d'Istanbul fut la réalisation des premières publications scientifiques en langue française rassemblées sous le titre de : Travaux du Séminaire de Romanologie romane.

Après le départ de Spitzer pour l'université John Hopkins de Baltimore (Ohio) en 1936, c'est Erich Auerbach qui prit la relève à la tête du département jusqu'en 1947.

De toute évidence il ne suffit pas de créer un département en prenant comme modèle le système universitaire moderne. Il faut également trouver des enseignants doués, capables d'assurer un enseignement au niveau international, respectant les méthodes et les acquis des sciences contemporaines. C'est surtout dans cette optique qu'il faut évaluer le rôle d'Auerbach. 

de langue et littérature françaises s'ancra à l'université d'Istanbul. En outre, les premiers académiciens turcs se formèrent sous sa direction pour assurer, sans aucune concession, le même enseignement scientifique jusqu'aux années 80 . mai 1942 et avril 1945.

L'objet de cet ouvrage, écrit-il, l'interprétation du réel à travers la représentation (ou « imitation ») littéraire, m'occupe déjà depuis très longtemps. Ce fut à l'origine la discussion qu'on trouve au Xème livre de la République - où Platon place la mimésis au troisième rang après la vérité - conjointement avec l'assertion de Dante selon laquelle la Comédie présente la réalité vraie, qui me servirent de point de départ. (...) Ainsi se développèrent un certain nombre d'idées directrices que je tentai de suivre ${ }^{3}$ (1968: 549 ).

Voici comment sa première assistante turque, Süheyla Bayrav, qualifie le livre de son maître dans un compte-rendu publié dans Dialogues:

Une grande pénétration psychologique, beaucoup de compréhension, des remarques subtiles, la connaissance approfondie et de longue date des matières traitées font les charmes de ce livre qu'on lit d'un bout à l'autre avec le même intérêt soutenu et que la majorité des critiques ont salué comme un grand livre (1949 : 140).

Ce paragraphe de Süheyla Bayrav reflète également les qualités d'Auerbach en tant qu'enseignant et témoigne de son impact dans la tradition académique du département. Plus de quatre cents mémoires de licence ont été faits par les étudiants jusqu'en 1982, l'année où une nouvelle réforme universitaire a changé le système complètement pour adopter le modèle des universités américaines. Les fondements théoriques de ces mémoires sont constitués par l'enseignement de Spitzer et d'Auerbach ainsi que de leurs prolongements ultérieurs, à savoir la linguistique structurale et la sémiotique greimassienne.

Un autre ouvrage d'Auerbach a eu également un sort intéressant : son Introduction aux études de philologie romane a été d'abord traduite en turc par S. Bayrav et publiée à Istanbul en 1944. Par la suite le texte original a été publié en français à Francfort en 1949 et sa version anglaise à New York en 1961 sous le titre de Introduction to Romance Languages and Littérature.

Avant son départ en 1947, Auerbach organisa la publication d'une revue scientifique : La revue des philologies occidentales. Après la fin de la II ${ }^{\mathrm{èm}}$ Guerre mondiale, presque tous les savants allemands y compris Auerbach, ont quitté la Turquie pour les États-Unis.

A l'époque où les enseignants d'origine allemande sont partis, le département comptait un nombre suffisant d'enseignants d'origine turque pour assurer le fonctionnement du département. Ils étaient tous disciples de Spitzer et d'Auerbach. Néanmoins, pendant cette période de l'après guerre, c'est le gouvernement français qui commença à envoyer régulièrement un professeur titulaire et des lecteurs agrégés pour une durée de deux ans. Ainsi Guy Michaud (1949-1950), Henri Bornecque, Langlade, et Greimas vinrent enseigner successivement à la Faculté des Lettres.

31 L'ambiance stimulante du département de langue et littérature françaises permit à Guy Michaud de publier son Introduction à une science de la littérature à Istanbul. En juin 1950, Michaud mit en exergue à son livre, qui est l'une des premières approches scientifiques du texte littéraire, l'« avertissement » suivant : 
L'occasion qui nous était offerte par la Faculté des Lettres de l'Université d'Istanbul de fixer par écrit l'essentiel d'un enseignement de plusieurs années a fait naître cet ouvrage avant terme. (...) Nous formons le vœu que, dans son état présent, cet ouvrage ne soit pas seulement une "introduction", mais qu'il soit jugé digne de susciter des critiques et des discussions, d'où pourrait naître ultérieurement une édition plus élaborée.

Ce vœu fut entendu puisque à la fin de la même année, il republia à nouveau l'étude en question aux éditions A.-G. Nizet, à Paris.

3 Parmi les professeurs français qui travaillèrent à l'Université d'Istanbul, c'est Greimas qui connut une notoriété nationale et internationale. En fait, Greimas avait été nommé en 1959 à l'Université d'Ankara, mais une fois tous les quinze jours il venait assurer des cours à la Faculté des Lettres de l'Université d'Istanbul. Et c'est d'ailleurs ici qu'il laissa le plus de traces.

Son séjour en Turquie conduisit Greimas à s'orienter vers une nouvelle recherche qui allait devenir plus tard la "sémiotique ${ }^{4}$." Voici comment il répond à la question : « Comment êtes-vous devenu sémioticien?»

Je crois que je suis devenu sémioticien - mais ceci ne devrait peut-être pas être généralisé - parce que je me suis trouvé abandonné à moi-même. Étant nommé à l'Université d'Alexandrie et à court de documents, au lieu de poursuivre mes recherches en lexicologie, comme c'était mon intention, j'ai été amené à réfléchir sur les conditions d'existence et les pratiques de la lexicologie, et d'une façon générale, sur les possibilités de l'analyse des significations. Donc, quand je suis arrivé chez vous, en Turquie, je n'étais déjà plus lexicologue, mais je n'avais pas encore de méthode plus sûre à vous proposer et je pensais qu'une pratique lexicologique était utile. (...)

Pendant mon séjour en Turquie, j'ai continué donc mes réflexions sur cette nouvelle façon d'aborder les problèmes de la signification, qui est devenue depuis la sémantique. Et c'est en effet les premiers résultats de mes recherches que j'ai soumis à mes étudiants turcs à Istanbul. J'ai repris ensuite les éléments de ce cours pour le refaire à Paris et le publier sous la forme de Sémantique structurale.

Comme pour la majorité des enseignants qui étaient de passage à Istanbul, pour Greimas aussi les cours avaient trouvé un écho parmi ses étudiants et collègues turcs. Ainsi la thèse de doctorat de son assistant de l'époque, Tahsin Yücel, inspira un chapitre de la Sémantique structurale intitulé « Un échantillon de description. »

Le maître de l'École sémiotique de Paris justifie son choix de la manière suivante :

Nous avons pensé qu'il ne serait pas inutile de présenter, au terme - provisoire - de ces réflexions, un échantillon de description à peu près complète, opérant à partir d'un corpus donné, rendant compte des procédures utilisées et proposant finalement les modèles définitifs d'organisation d'un micro-univers sémantique. Nous avons choisi, dans cette intention, l'univers de Georges Bernanos. L'exemple s'est pratiquement imposé à nous, du fait de l'existence de l'étude de Tahsin Yücel, l'Imaginaire de Bernanos, récemment présentée, sous forme de thèse de doctorat, devant la Faculté des lettres d'Istanbul (Greimas 1966 : 222).

Pour conclure, je laisserai la parole encore une fois, au grand théoricien de la sémiotique. Je souhaiterais que ses paroles reflètent également les sentiments de tous les grands maîtres de notre domaine qui, par leurs efforts et dévouement, nous ont laissés à l'Université d'Istanbul, un terrain fertile, favorable à la recherche scientifique :

(...) ces quelques années passées en Turquie ont marqué ma vie. J'y ai trouvé une atmosphère propice à la réflexion et, surtout à Istanbul, un milieu universitaire stimulant et accueillant issu de la Réforme d'Atatürk et grandi au contact de la pensée des grands maîtres de linguistique et de littérature. J'ai trouvé en Turquie 
également des amis, tel le professeur Nusret Hizir, élève lui-même du grand logicien Reichenbach, qui a su m'initier aux problèmes fondamentaux de la logique symbolique. C'est dire que mon séjour en Turquie peut être caractérisé en termes d'échanges et de dons réciproques. (Vardar, $1976: 30$ )

\section{BIBLIOGRAPHIE}

AUERBACH, Erich (1946) : Mimesis : Dargestellte Wirklichkeit der abendländischen Literatur von Erich Auerbach. Berne, A. Francke-Verlag.

AUERBACH, Erich (1968) : Mimesis, La Représentation de la realité dans la littérature occidentale, (trad. fr. Cornélius Heim). Paris, Editions Gallimard.

BAYRAV, Süheyla (1949) : « Mimesis : Dargestellte Wirklichkeit der abendländischen Literatur von Erich Auerbach. Berne, A. Francke-Verlag, $1946 »$, compte-rendu, Dialogues, nº 1, Publication de la Faculté des Lettres d'Istanbul - Institut de Philologie française et romane, Istanbul.

Dialogues (1949) : publication de la Faculté des Lettres d'Istanbul, Institut de philologie française et romane, $\mathrm{N}^{\circ} 1$.

GREIMAS, Aljirdas-Julien (1966) : Sémantique structurale. Paris, Larousse.

KLEMPERER, Victor (1948): « Philologie im Exil », in Aufbau Kulturpolitische Monatsschrift. Berlin, Heft 10.

MICHAUD, Guy (1950) : Introduction à une science de la littérature. Université d'Istanbul, Pulman Matbaasi.

MICHAUD, Guy (1950) : (avec la collaboration de E. Fraenkel) : Introduction à une science de la littérature. Paris, Editions A.-G. Nizet.

STAROBINSKY, Jean (1970): « Leo Sptizer et la lecture stylistique », in Études de style de L. Spitzer. Paris, Gallimard.

VARDAR, Berke (1976): (propos recueillis par) : « Entretien avec A.-J. Greimas », Dilbilim 1. Revue du Département de français de l'École supérieure des langues étrangères de l'Université d'Istanbul.

WILDMANN, Horst (1999) :Exil und Bildungshilfe, trad, en turc par A. Kazancigil et S. Bozkurt sous le titre de Atatürk ve Universite Reformu (Atatürk et la Réforme universitaire), Istanbul. Kabalci yay.

\section{NOTES}

1. qui a précédé l'actuel département de langue et littérature françaises.

2. Dialogues (1949) : publication de la Faculté des Lettres d'Istanbul, Institut de philologie française et romane, $\mathrm{N}^{\circ} 1$, « Présentation » du premier numéro.

3. Ibid., traduction française, "Postface", p. 549. 
4. En l'année 1961-1962, Greimas fait un cours de « sémantique structurale » à la Faculté des Lettres d'Istanbul.

\section{RÉSUMÉS}

Dans cet article l'auteur se propose de raconter la création du département de langue et littérature françaises à la Faculté des Lettres de l'Université d'Istanbul. Créé en 1933 par Léo Spitzer, le département en question fut dirigé de 1936 à 1947 par Erich Auerbach et toute une équipe d'enseignants d'origine juive qui avaient fui le régime nazi en Allemagne et avaient été accueillis par la Turquie. Après la 2ème guerre mondiale, des professeurs comme Michaud, Bornecque et Greimas enseignèrent dans ledit département laissant des traces favorables à la recherche scientifique.

In this article the author sets out to relate the foundation of the department of French language and literature at the Faculty of Arts of Istanbul university. This department was created in 1933 by Leo Spitzer, then was run from 1936 until 1947 by Erich Auerbach, along with a teaching staff of Jewish origin who had tied the Nazi regime in Germany and been welcomed by Turkey. After the 2nd World War such professors as Michaud, Bornecque and Greimas lectured in this department opening new paths to further scientific research.

\section{INDEX}

Mots-clés : Turquie, Université d'Istanbul, Spitzer, Auerbach, Michaud, Bornecque, Greimas Keywords : Turkey, Université d'Istanbul, Spitzer, Auerbach, Michaud, Bornecque, Greimas

\section{AUTEUR \\ OSMAN SENEMOGLU}

Université d'Istanbul 